\title{
Bubbles and Crashes with Partially Sophisticated Investors*
}

\author{
Milo Bianchi ${ }^{\dagger} \quad$ Philippe Jehiel ${ }^{\ddagger}$
}

September 29, 2010

\begin{abstract}
We analyze bubbles and crashes in a model in which some investors are partially sophisticated. While the expectations of such investors are endogenously determined in equilibrium, these are based on a coarse understanding of the market dynamics. We highlight how such investors may endogenously switch from euphoria to panic and how this may lead to equilibrium bubbles and crashes even in a purely speculative market in which information is complete and it is commonly understood that the bubble cannot grow forever. We also show how this setting can match stylized empirical facts, and we investigate whether bubbles may last longer when the share of fully rational traders increases.
\end{abstract}

Keywords: Speculative bubbles, crashes, bounded rationality.

JEL codes: D84, G12, C72.

*This is a substantially revised version of Bianchi and Jehiel (2008). We thank numerous seminar participants and in particular Cedric Argenton, Abhijit Banerjee, Markus Brunnermeier, Mike Burkart, Sylvain Chassang, Gabrielle Demange, Daniel Dorn, Tore Ellingsen, Guy Laroque, Jacques Olivier, Marco Ottaviani, Peter Raupach, Marcus Salomonsson, Jean-Marc Tallon, Johan Walden, Jörgen Weibull, Muhamet Yildiz and Robert Östling for useful discussions. Milo Bianchi gratefully acknowledges financial support from Region Ile-de-France and from the Risk Foundation (Groupama Chair). Philippe Jehiel thanks the European Research Council for support.

${ }^{\dagger}$ University Paris Dauphine. E-mail: milo.bianchi@dauphine.fr

${ }^{\ddagger}$ Paris School of Economics and University College London. E-mail: jehiel@enpc.fr 


\section{Introduction}

Speculative bubbles seem widespread (see Garber, 1990; Kindleberger, 2005) but their foundations remain largely unclear. By definition, in speculative bubbles, investors are willing to pay high prices today only because they expect the selling price to be even higher in the future (Stiglitz, 1990). This is typically inconsistent with rational expectations, at least when prices cannot grow without bounds (Tirole, 1982). Speculative bubbles have often been derived in the literature by assuming that some agents are either mechanical in their trading decisions or that they hold exogenously given (typically optimistic) expectations. ${ }^{1}$

This paper takes a different approach. In our model, the expectations of all agents are endogenously determined and investment strategies are decided optimally given expectations. Agents are neither overconfident nor optimistic. But while some agents are fully rational in the sense of having correct expectations, others are less sophisticated and form their expectations based on some coarse understanding of the working of the market. An important contribution of this paper is to show that bubbles and crashes can endogenously emerge in such contexts. In the analysis, we will highlight how the expectations of partially sophisticated agents evolve along the life cycle of the speculative market giving rise first to bubbles and then to crashes, and we will suggest how the qualitative features of our equilibrium match the intuitive wisdom about bubbles.

We develop our main ideas in a simple setting intended to be the least favorable to bubbles. We consider a deterministic market with complete information in which agents can trade an asset with no fundamental value. Importantly, traders are heterogeneous in their ability to understand others' trading strategies, and thus in their expectations about the market dynamics.

We consider two extreme types of investors: fully rational investors who have rational expectations, as usual; and partially sophisticated investors who design their investment strategies based only on aggregate statistics about

\footnotetext{
${ }^{1}$ There is also a vast literature with overlapping generations in which bubbles allow for capital transmission from one generation to the next. That viewpoint about bubbles is complementary to the one developed here that views bubbles as purely speculative.
} 
market dynamics. These statistics will be formally defined later on, but it is worth emphasizing from now that in our model all agents, irrespective of their degree of sophistication, have enough understanding of the situation to realize that the market is overvalued and bound to crash (indeed, this is common knowledge). We note that such an understanding is in line with the observed evidence that, at least eventually, most agents realize they are in a speculative market (see Shiller, 1989). ${ }^{2}$ Trade is then driven by the fact that all agents believe, rightly or wrongly, that they can take advantage of investing in the speculative market and exiting at the right time.

The key mistake our partially sophisticated investors make is in failing to understand when, based on commonly observed history of trades, other investors exit the market. ${ }^{3}$ Such a mistake is due to these investors' partial understanding of the market dynamics. In fact, in equilibrium, the statistics employed by partially sophisticated investors match the actual market dynamics averaged over time, but they are assumed not to have access to more precise statistics relating the market dynamics to the life cycle of the bubble.

We view the knowledge of the various types of investors as the result of historical learning whereby, when facing a new bubble episode, investors form their optimal strategies based on their knowledge about similar past bubble episodes. Whether this knowledge is based on more or less detailed statistics determines investors' sophistication.

The basic mechanism which generates bubbles and crashes in our framework is given by the resulting interaction between investors' historical knowledge and their observation of market trends in the current bubble episode (in

\footnotetext{
${ }^{2}$ Shiller (1989) reports that just before the U.S. stock market crash of October 1987, $84 \%$ of institutional investors thought that the market was overpriced; $78 \%$ of them thought that this belief was shared by the rest of investors and, still, $93 \%$ of them were net buyers. There are also several anecdotes about this. For example, Eric Janszen, a leading commentator of speculative phenomena, wrote in the middle of the Internet bubble (November 1999): "During the final stages, the mania participants finally admit that they are in a mania. But they rationalize that it's OK because they - only they and not the other participants - will get out in time." (article accessible at www.bankrate.com)

${ }^{3}$ This assumption may echo the observation that the day of the crash often appears to be quite similar to many other days. Even the systematic analysis by Cutler, Poterba and Summers (1989) concludes that "many of the largest market movements in recent years have occurred on days when there were no major news events."
} 
line with the experimental evidence in Haruvy, Lahav and Noussair, 2007). Partially sophisticated investors know the market dynamics averaged along days in which most people want to buy and days in which most people want to sell. As a result, they get a positive surprise after any "good day" and a negative surprise after any "bad day". Specifically, along our bubble equilibrium, investors first observe a series of rising prices. Partially sophisticated investors interpret such unexpectedly high prices as a sign that the price can go even further. ${ }^{4}$ In doing so, they end up remaining invested for too long. Such investment strategies are in turn exploited by fully rational investors, who feed the bubble for a while and exit just before the (endogenous) crash. Upon observing the massive sale by rational investors, partially sophisticated investors realize it is time to sell and this indeed leads to the crash.

In our main analysis, we define the structure of bubble equilibria in terms of investment strategies and expectation dynamics (as sketched above), we characterize the conditions for the existence of bubble equilibria, and we analyze how the maximal duration of bubbles varies as a function of our parameters, in particular the share of rational investors and how new investors are attracted as a function of the history of prices. We also discuss our findings in relation to stylized empirical evidence about bubbles and crashes.

The rest of the paper is organized as follows. The next subsection reviews some of the key features of our model and discusses the related literature, highlighting how the mechanism behind our bubble equilibrium differs from existing ones. Our main model is presented in Section 2 and the analysis of equilibrium strategies and expectations are derived in Section 3. In Section 4 , we extend our setting by considering the case of uncertainty averse agents, and we show that whether rational investors have a stabilizing role depends on the attitude of investors toward uncertainty. In Section 5, we relate our findings to empirical evidence; we elaborate further on the interpretation of our equilibrium concept and discuss the robustness of our results in richer settings. Omitted proofs are provided in the Appendix.

\footnotetext{
${ }^{4}$ This captures a strong regularity documented in Shiller (2000). As the price increases, more people display "bubble expectations", i.e. the belief that, despite the market being overvalued, it will still increase for a while before the crash.
} 


\subsection{Related literature}

There is a vast literature on speculative bubbles, and we only review some general themes here. ${ }^{5}$ First, our approach differs from a good part of the literature in behavioral finance in which non-fully rational investors are modeled in an exogenous fashion (with no consistency requirement) and the focus is on how rational investors would behave in such environments (Shleifer, 2000). In particular, an important strand of this literature focuses on the effects of purely mechanical traders (De Long, Shleifer, Summers and Waldmann, 1990a) or of agents who form their expectations about future prices simply by extrapolating from past market trends (Cutler, Poterba and Summers, 1990 and De Long, Shleifer, Summers and Waldmann, 1990b). As emphasized above, our equilibrium approach allows agents to have enough sophistication to understand that they are in a bubble and that the market must crash (as opposed to extrapolative agents, who in a phase of rising prices would expect the prices to increase with no bounds). Moreover, as detailed in Section 4, we differ from this literature in that the potential destabilizing character of rational agents in our setup can only arise when agents are ambiguity averse.

A second important strand of the literature on speculative bubbles builds on the fact that some information, e.g. about the value of fundamentals, is dispersed among agents and that agents hold subjective priors. ${ }^{6}$ We differ from this literature first as we consider a setting with complete information. This allows us to highlight that bubbles in our setup are caused by the partial sophistication of some traders rather than by the presence of asymmetric information; also, this can be used to shed new light on the substantial experimental evidence that bubbles emerge even in contexts in which the structure of the game is commonly known to subjects (see Porter and Smith, 2003, for a review). Second, while similarly to approaches with subjective

\footnotetext{
${ }^{5}$ For a more detailed review, see e.g. Brunnermeier (2007).

${ }^{6}$ From a theoretical viewpoint, private information alone cannot explain bubbles, as can be inferred from the no-trade theorems (Milgrom and Stokey, 1982). Somewhat more generally, Allen, Morris and Postlewaite (1993) show that bubbles may arise in a finite setting with private information only if one also introduces ex-ante inefficiency, short sale constraints, or lack of common knowledge of agents' trades. See, in particular, Morris (1996) and Biais and Bossaerts (1998) for models of speculation with subjective priors.
} 
priors we model agents with possibly erroneous expectations, an important difference is that the perceptions of our boundedly rational agents derive from correct (yet coarse) understanding of others' strategies. There is no such constraint in the approaches with subjective priors, in which the subjective beliefs are left exogenous. ${ }^{7}$

It should also be mentioned that in contrast with our approach, the literature has typically modeled bubbles and crashes separately. For example, Gennotte and Leland (1990) focus on the role of hedge funds in provoking the crash while taking as given the fact that the market is overvalued. Abreu and Brunnermeier (2003) focus on how coordination issues among rational arbitrageurs may delay the crash, while abstracting from the underlying process generating the bubble. On the other hand, De Long, Shleifer, Summers and Waldmann (1990b) explain how feedback trading can generate a bubble, but exogenously impose an end period at which the crash occurs. Scheinkman and Xiong (2003) show how overconfidence can sustain speculative trade but do not consider how the crash may endogenously occur. ${ }^{8}$

Finally, by emphasizing the role of cognitive heterogeneity, our work is related to a wide literature on the limits to information processing. ${ }^{9}$ In particular, our idea of equilibrium is in the spirit of Jehiel (2005) who assumes, in the context of extensive form games, that each player understands only the aggregate behavior of his opponents over a bundle of nodes/states. ${ }^{10}$ Our

\footnotetext{
${ }^{7}$ It may be argued that what is left exogenous in our setting is the fineness of the statistical knowledge of agents. More work is required to assess empirically what kind of statistical knowledge is available in practice. From a theoretical viewpoint, however, our results hold for a broader class of cognitive types (see Section 5.3).

${ }^{8}$ A notable exception is Allen and Gale (2000) who provide a model in which bubbles arise as debt-financed investors are willing to pay high prices for a risky asset and crashes occur when uncertainty in the real or in the financial sector resolves. Our model abstracts both from uncertainty and agency problems in generating bubbles and crashes.

${ }^{9}$ See in particular Higgins (1996) for an exposition of the idea of accessibility in psychology and Kahneman (2003) for economic applications. Many authors have explored such themes in strategic interactions (see Rubinstein, 1998 and the references therein and Jehiel, 1995; Jehiel, 2005; Jehiel and Samet, 2007), and financial markets (see Hirshleifer, 2001).

${ }^{10} \mathrm{~A}$ related idea is developed in static games of incomplete information by Eyster and Rabin (2005). Alternative and complementary approaches to partial sophistication include Nagel (1995), Stahl and Wilson (1995), and Camerer, Ho and Chong (2004). These approaches differ from ours in that they focus on initial play whereas we focus on behaviors
} 
model can be viewed as providing a finance theoretical analog of such concepts and it allows us to shed new light on the emergence of bubbles and crashes and on the stabilizing role of arbitrageurs.

\section{Model}

Consider a market where an asset of fundamental value $p_{0}$ is traded. In each date $t$, a new population of cash-holders $K_{t}$ is attracted to the market, possibly as a function of the entire history of prices. ${ }^{11}$ Cash-holders have one unit of wealth and, upon arrival, they decide whether or not to buy the stock by submitting a demand schedule. ${ }^{12}$ It is assumed that neither borrowing nor short-selling is possible. Let $\beta_{t}$ denote the fraction of cash-holders who decide to buy. The amount of buy order at $t$ is then given by:

$$
B_{t}=\beta_{t} K_{t}
$$

Sell orders are due either to deliberate selling or to liquidity shocks. More precisely, we denote by $X_{t}$ the amount of outstanding stocks at date $t$, which may vary with $t$ (e.g. due to new stocks becoming tradable as lock-up periods expire). ${ }^{13}$ In each period, with probability $z$, a stock holder is hit by a liquidity shock which leads him to sell immediately and to stay out of the market from then on. Denote by $\sigma_{t}$ the fraction of stock-holders who decide to sell. The amount of sell orders (including the exogenous supply of stocks $z X_{t}$ induced by liquidity shocks) is then given by:

$$
S_{t}=\sigma_{t} X_{t}
$$

that would persist even after (imperfect) learning has taken place.

${ }^{11}$ See Barber and Odean (2008) for empirical evidence on which dynamics of prices and trade volumes tend to attract investors' attention.

${ }^{12}$ In Section 5.3, we briefly discuss other trading strategies such as limit or stop-loss orders.

${ }^{13}$ One may alternatively allow the firm to deliver new stocks, in which case the following analysis would go through with minor notational changes. 
The price $p_{t}$ in period $t$ is determined by a standard market clearing condition, or more precisely a cash-in-the-market pricing (Allen and Gale, 1994), that is,

$$
p_{t}=\frac{B_{t}}{S_{t}}
$$

In each period, each investor chooses an investment strategy that maximizes his expected payoff. The payoff to an individual who buys a stock at time $t$ and sells it at time $s$ is simply $\left(p_{s}-p_{t}\right)$. That is, we ignore discounting and risk aversion, and investment in our economy can only be driven by the belief that the selling price will be higher in the future. ${ }^{14}$

Beliefs about future prices are based both on the observation of the history of trades and on the expectation about other agents' strategies. The history of trades is public, so that agents share the same information. Moreover, the way in which $K_{t}$ and $X_{t}$ depend on the history of trades is deterministic and well-understood by everyone (specific functional forms will be assumed later on, whenever needed). Hence, for a given history of trade, expected price dynamics are fully determined by the expectations about other agents' strategies. As already mentioned, investors differ in how they form their expectations about others' strategies, which corresponds to different levels of sophistication $\theta$ and typically results in different expectations about future prices.

Formally, denote by $p_{t}^{\theta, s}\left(H^{s}, \Sigma^{\theta, s}\right)$ the expectation made at the start of period $s$ by an agent $i \in \theta$ about the price at $t$, where $t \geq s$ (a similar notation is employed for the other endogenous variables). This expectation depends on the commonly observed history of trades $H^{s}=\left(p_{1}, p_{2}, \ldots, p_{s-1}\right)$ and on type-specific expectations about future investment strategies $\Sigma^{\theta, s}=$ $\left(\beta_{s}^{I, s}, \sigma_{s}^{\theta, s}, \beta_{s+1}^{I, s}, \sigma_{s+1}^{\theta, s}, \ldots, \beta_{t}^{I, s}, \sigma_{t}^{\theta, s}\right)$. To illustrate our ideas, we consider a situation with two cognitive types: fully rational investors $R$, in proportion $r$, and partially sophisticated agents $I$, in proportion $(1-r) .{ }^{15}$ (These proportions apply both to new investors with cash and with stock.) Rational agents

\footnotetext{
${ }^{14}$ Introducing discounting would not alter the main conclusions of the paper and it would make the analysis a little more cumbersome. Ambiguity aversion is briefly considered in Section 4 .

${ }^{15}$ In Section 5 we briefly discuss the case with a richer set of cognitive types.
} 
understand perfectly well the patterns of other investors' strategies. Hence, denoting by $\beta_{t}$ and $\sigma_{t}$ the actual buy and sell rates arising in equilibrium, their expectations must satisfy

$$
\beta_{t}^{R, s}=\beta_{t} \text { and } \sigma_{t}^{R, s}=\sigma_{t} \text { for every } t \geq s .
$$

Partially sophisticated agents, on the other hand, expect constant buy and sell rates throughout the duration of the speculative market, where these rates coincide with the actual aggregate intensities averaged over time. Specifically, denote by $t=1$ the first date in which the trading price exceeds the fundamental price $p_{0}$ (the start of the bubble) and by $t=T+1$ the first date in which the price goes back to the fundamental (the end of the bubble), as determined in equilibrium. The average buy rate $\bar{\beta}$ and the average sell rate $\bar{\sigma}$ for the sequence of buy and sell decisions arising in equilibrium are

$$
\bar{\beta}=\frac{1}{T+1} \sum_{t=1}^{T+1} \beta_{t} \text { and } \bar{\sigma}=\frac{1}{T+1} \sum_{t=1}^{T+1} \sigma_{t} .
$$

I-agents' expectations are required to correspond to these averages. Hence, we have

$$
\beta_{t}^{I, s}=\bar{\beta} \text { and } \sigma_{t}^{I, s}=\bar{\sigma} \text { for every } t \geq s .
$$

Based on $H^{s}$ and $\Sigma^{\theta, s}$, an agent of type $\theta$ forms expectations about the price in period $t \geq s$. Based on these expectations, he can design his optimal investment strategy.

Definition 1 (Equilibrium): An investment strategy profile is an equilibrium if, all along the equilibrium path, each agent's investment strategy maximizes his expected payoff, given the expectations defined in equations (2), (3) and (4). 


\section{Analysis}

\subsection{Equilibrium strategies and expectations}

Since agents are risk neutral, once they decide to trade, they trade their entire holding of stock or cash. Optimal trading strategies at time $t$ can be expressed simply as a function of the expected prices at $t$ and at $t+1$ given the information available at the start of $t$. An agent $i \in \theta$ prefers to buy at $t$ if and only if

$$
p_{t+1}^{\theta, t} \geq p_{t}^{\theta, t}
$$

Condition (5) is sufficient since if $p_{t+1}^{\theta, t} \geq p_{t}^{\theta, t}$ the strategy of buying at $t$ and selling at $t+1$ would deliver positive payoffs. Condition (5) is also necessary since if $p_{t+1}^{\theta, t}<p_{t}^{\theta, t}$ buying at $t$ would be dominated by buying at $t+1 .{ }^{16}$ The same argument applies to any agent $j \in \theta$ with stocks who decides whether to keep it or sell it.

We focus on bubble equilibria of the following form. In each period $t \leq$ $T-1$ everyone buys and only liquidity sellers (in proportion $z$ ) sell; in period $T, I$-investors buy and $R$-investors sell; at $T+1$, everyone tries to sell but no one is willing to buy. ${ }^{17}$ The resulting equilibrium buy and sell rates are

$$
\left(\beta_{t}, \sigma_{t}\right)=\left\{\begin{array}{cl}
(1, z) & \text { for } t \leq T-1 \\
(1-r, z+r(1-z)) & \text { for } t=T \\
(0,1) & \text { for } t=T+1
\end{array}\right.
$$

Given the expressions of $\beta_{t}$ and $\sigma_{t}$ in (6), and the consistency condition (3), $I$-agents are induced to expect the buy and sell rates to be

$$
\bar{\beta}=\frac{T-r}{T+1},
$$

\footnotetext{
${ }^{16}$ Without loss of generality, we assume that when indifferent an individual invests.

${ }^{17}$ Intuitively, trade occurs either among people with different needs, as described by the liquidity sellers, or among those with different expectations, as described by the different types.
} 
and

$$
\bar{\sigma}=\frac{T z+r(1-z)+1}{T+1},
$$

respectively. Together with the form of $K_{t}$ and $X_{t}$, expectations over the buy and sell rates translate into expectations about future prices.

For this to be an equilibrium, we need that, up to $T, I$-agents expect the price to increase in the next period and that at $T+1$ they expect the price to drop at $T+2$. That is,

$$
p_{t+1}^{I, t} \geq p_{t}^{I, t} \text { for each } t \leq T
$$

and

$$
p_{T+2}^{I, T+1}<p_{T+1}^{I, T+1} .
$$

Moreover, for $R$-agents to find their strategy optimal, the price must be increasing up to $T$ and it must be dropping at $T+1$. Thus,

$$
p_{t} \geq p_{t-1} \text { for each } t \leq T
$$

and

$$
p_{T+1}<p_{T}
$$

At this point in order to explore further the existence and properties of bubble equilibria, we need to specify functional forms for $K_{t}$ and $X_{t} \cdot{ }^{18}$ While the amount of new investors may depend on the entire history of prices and expectations, we simplify the analysis by assuming that $K_{t}$ depends only on $p_{t-1}$ and $p_{t-2}$; in particular, more investors are attracted when $p_{t-1}$ is higher and the marginal effect of an increase in $p_{t-1}$ is higher when $p_{t-1}$ exceeds $p_{t-2}$. The idea that higher prices yesterday attract more investors has been documented by Barber and Odean (2008). The relation with $p_{t-2}$ may be interpreted viewing $p_{t-2}$ as a reference point for $p_{t-1}$ so that when $p_{t-1}>p_{t-2}$ more investors are attracted at $t$ as a function of $p_{t-1}$ than when

\footnotetext{
${ }^{18}$ In Section 5.3, we discuss the sensitivity of our results to different functional forms.
} 
$p_{t-1}<p_{t-2} \cdot{ }^{19}$ Specifically, we assume that, for $t>1$,

$$
K_{t}\left(p_{t-1}, p_{t-2}\right)=\left\{\begin{array}{lll}
\alpha_{H} p_{t-1} & \text { if } & p_{t-1}>p_{t-2} \\
\alpha_{L} p_{t-1} & \text { if } & p_{t-1} \leq p_{t-2}
\end{array}\right.
$$

where $\alpha_{L} \leq \alpha_{H}$. To complete the description, we exogenously set $p_{t}=p_{0}=0$ for $t \leq 0$ and $K_{1}=K$, with $K>0$.

Moreover, we capture the idea that in bubble episodes speculative stocks tend to be progressively released in the market (Cochrane, 2002; Hong, Scheinkman and Xiong, 2006) by assuming that a mass $X$ of stock becomes tradable (exogenously) in each period. The amount of outstanding stocks in $t$ is then

$$
X_{t}=t X
$$

We assume the functions (13) and (14) are commonly known and well understood by all investors. Accordingly, we have:

$$
p_{t}^{I, t}=\frac{\bar{\beta} K_{t}\left(p_{t-1}, p_{t-2}\right)}{\bar{\sigma} X_{t}}
$$

and

$$
p_{t+1}^{I, t}=\frac{\bar{\beta} K_{t+1}\left(p_{t}^{I, t}, p_{t-1}\right)}{\bar{\sigma} X_{t+1}} .
$$

The strategies defined above constitute a bubble equilibrium if conditions (9), (10), (11), (12) are satisfied. We now analyze how these conditions define the equilibrium duration of the bubble $T$.

\subsection{Equilibrium duration of a bubble}

We first observe that irrespective of the buying and selling strategies of investors, bubbles must burst in finite time. Even if $\left(\beta_{t}, \sigma_{t}\right)=(1, z)$ for all $t$,

\footnotetext{
${ }^{19}$ Viewing $p_{t-2}$ as a reference point for $p_{t-1}$ is reminiscent of prospect theory (see Kahneman and Tversky, 1979). Alternative models would allow agents' expectations to affect the reference point (as in Koszegi and Rabin, 2006). Our formulation allows for a simpler analysis even if we expect similar insights with these alternative models of reference points.
} 
$p_{t+1} \geq p_{t}$ requires

$$
t+1 \leq \frac{\alpha_{H}}{z X} \equiv T_{0},
$$

thereby making of $T_{0}$ an upper bound on the duration of bubbles. However, the equilibrium duration of a bubble will typically be much smaller than $T_{0}$.

The maximal duration of the bubble is defined by two conditions (we only sketch the analysis here and leave a more detailed derivation for Section 6.1 in the Appendix). The first is condition (9), which must hold in particular for $t=T$. This writes

$$
T+1 \leq \frac{\bar{\beta}}{\bar{\sigma}} \frac{\alpha_{H}}{X} \equiv W(T)
$$

where $\bar{\beta}$ and $\bar{\sigma}$ are defined in (7) and (8). Defining $T_{1}$ as the largest $T$ such that $T+1=W(T)$ (assuming for now it exists), it can be shown with simple algebra that condition (9) defines an upper bound on $T$, e.g.

$$
T \leq T_{1}
$$

This upper bound comes from the fact that, as time goes by, $I$-investors perceive that the selling pressure increases more than the buying capacity. This implies that rational investors cannot sell too late otherwise $I$-investors would not buy.

A second upper bound on $T$ is defined by condition (11). In particular, (11) must hold for $t=T$, which requires

$$
T \leq \frac{(1-r) \alpha_{H}}{(z+r(1-z)) X} \equiv T_{2}
$$

The upper bound $T_{2}$ comes from the fact that, since rational investors accumulate stocks as the bubble grows, they cannot wait too long before selling or they would not find enough irrational investors to absorb the selling pressure. We can then define the largest $T$ that can be sustained in equilibrium, denoted by $T_{\max }$, as the minimal $T$ satisfying both condition (16) and (17), i.e.

$$
T_{\max } \equiv \min \left\{T_{1}, T_{2}\right\}
$$


We also notice that in equilibrium the bubble cannot be too short either. This is due to the requirement that $I$-investors should sell at $T+1$, as expressed by condition (10), and so that the buying capacity at $T+1$ should not be too large. As shown in Section 6.1, this in turn imposes a lower bound on $T$ whereby rational investors cannot sell too early otherwise $I$-investors would not sell at $T+1$ and the market would not crash (so $R$-agents would make more profit by selling later than at $T$ ). Finally, it can easily be shown that if $I$-investors sell at $T+1$ they do not buy at any later point in time, so $R$-investors have no incentive to buy at $T+1$. This implies $\beta_{T+1}=0$ and so $p_{T+1}=p_{0}=0$. Hence, if condition (10) is satisfied, condition (12) follows.

We can now characterize when a $T \geq 1$ satisfying conditions (9)-(12) exists as a function of the exogenous parameters $\alpha_{H}, \alpha_{L}, X, z$ and $r$. As in standard models, we cannot have bubbles if all investors are fully rational. In particular, $r$ has to be small enough so that all rational agents are able to sell at $T$, which defines the constraint (17). As we must have $T_{2} \geq 1$, we need

$$
r \leq \frac{\alpha_{H}-z X}{\alpha_{H}-z X+X} \equiv r_{\max }
$$

Hence, we can define a necessary condition for the existence of a bubble equilibrium.

Proposition 1 There exists a $r_{\max }<1$ such that if $r>r_{\max }$, then no bubble equilibrium exists.

We then provide sufficient conditions for the existence of a bubble equilibrium, characterizing in particular when the bubble duration $T_{\max }=\min \left\{T_{1}, T_{2}\right\}$ can be sustained in equilibrium. Again, we only sketch the intuition here and leave a more formal analysis to the Appendix (Section 6.1). Suppose first that $T_{1}<T_{2}$, which is the case when $r$ is sufficiently small. ${ }^{20}$ Since by definition $T_{1}$ is the latest date at which $I$-investors buy, they sell at $T_{1}+1$, as required by condition (10). Hence, conditions (9)-(12) would be satisfied at $T_{1}$ and we only need to make sure that $T_{1} \geq 1$. Given (15), a sufficient condition for having $T_{1} \geq 1$ is that $W(T)$ and $T+1$ intersects once for $T \geq 1$,

\footnotetext{
${ }^{20}$ See Section 6.2 for a more detailed characterization.
} 
which is the case when $W(1) \geq 2$. This writes

$$
\alpha_{H} \geq 2 X\left(z+\frac{1+r}{1-r}\right) \equiv \alpha_{H}^{*}(r, z, X)
$$

where it is easy to see that $\alpha_{H}^{*}$ increases in $X, r$ and $z$. The reason why a sufficiently large $\alpha_{H}$ is required for the existence of a bubble equilibrium is that the amount of new investors entering in each period is then sufficiently large to induce $I$-investors to buy for a while.

If instead $T_{2}<T_{1}$, conditions (9)-(12) would be satisfied if $I$-investors sell at $T_{2}+1$. This writes

$$
\alpha_{L}<\frac{\bar{\sigma}}{\bar{\beta}}\left(T_{2}+1\right) X \equiv \alpha_{L}^{*}(r, z, X)
$$

where $\bar{\sigma}$ and $\bar{\beta}$ are defined at $T_{2}$ and one can easily show that $\alpha_{L}^{*}>0$ and $\alpha_{L}^{*}<\alpha_{H}$. The reason why a sufficiently small $\alpha_{L}$ is required for a bubble to emerge is that when $T_{2}$ is smaller than $T_{1}, I$-investors would not sell at $T_{2}+1$ unless the drop in the entry of new investors is sufficiently large. Since by definition $p_{T_{2}}=p_{T_{2}-1}$, by (13) we would have $K_{T_{2}+1}=\alpha_{L} p_{T_{2}}$ and for a large drop in $K_{t}$ we would need $\alpha_{L}$ sufficiently small. ${ }^{21}$

More complete results are expressed in the following Proposition, which shows that bubble equilibria can emerge when $\alpha_{H}$ is large enough and when $\alpha_{L}, X, z$ and $r$ are small enough.

Proposition 2 If $\alpha_{L}<\alpha_{L}^{*}$ and $\alpha_{H} \geq \alpha_{H}^{*}$, then the above investment strategies with $T=T_{\max }$ define a bubble equilibrium. Moreover, $T_{\max }$ increases in $\alpha_{H}$ and decreases with $X, z$ and $r$.

\subsection{Expectations dynamics}

We can now illustrate how investors' expectations evolve along a bubble equilibrium with maximal duration as characterized by $T=T_{\max }$. Fully

\footnotetext{
${ }^{21}$ We are here ignoring integer problems and assume that $T_{2}$ is indeed an integer. If this were not the case, then we would require $R$-agents to randomize and sell at $T_{2}$ and $T_{2}-1$ so to make sure that indeed $p_{T_{2}}=p_{T_{2}-1}$. This would slightly change equation (6) without changing the substance of our analysis.
} 
rational agents have rational expectations, and so their expectation about the price dynamics is unchanged (and correct) throughout the duration of the bubble. As far as $I$-agents are concerned, their expectations may typically change once they observe the actual realized prices. More precisely, after any $t<T, I$-agents revise their expectation about the future prices upwards. This is because, in all these periods, $\beta_{t}>\bar{\beta}$ and $\sigma_{t}<\bar{\sigma}$ and so the realized prices are higher than expected. An higher price attracts more investors and, for a given expectation about future buy and sell rates, this allows prices to be even higher in the future. On the other hand, upon observing the realized price at $T, I$-agents revise their expectation about the future prices downwards whenever the realized price is lower than expected, which happens whenever $\beta_{T}<\bar{\beta}$ and $\sigma_{T}>\bar{\sigma}$. This requires that $r$ be not too small. Indeed, with simple algebra, it can be shown that

$$
p_{T+1}^{I, T+1}<p_{T+1}^{I, T} \Leftrightarrow \operatorname{Tr}>1,
$$

where the condition $\operatorname{Tr}>1$ implicitly defines a minimum $r_{1}>0$ such that $\operatorname{Tr}>1$ only if $r>r_{1}$ (see Section 6.2 for the derivation). Hence, if rational investors are sufficiently many, their massive sale at $T$ is bad news for the market as it makes $I$-investors realize that the prices will indeed be lower than expected.

$I$-investors' expectation about the date of the crash, instead, remains unchanged for $t<T$. This is due to the fact that, as long as $p_{t-1}>p_{t-2}$, $K_{t}$ is a linear function of $p_{t-1}$. As shown in equation (21), this makes $I$ investors' strategy insensitive to the current price realization. ${ }^{22}$ A change in their expectation about the date of the crash may instead occur when the amount of new investors entering the market at $T+1$ is very low, that is, when $p_{T}=p_{T-1}$ and $\alpha_{L}$ is sufficiently low. For $p_{T}=p_{T-1}$, we need that $T=T_{2}$ and so $T_{2}<T_{1}$ (otherwise $T_{2}$ could not be sustained as an equilibrium). The condition $T_{2}<T_{1}$ writes

$$
W\left(T_{2}\right)>T_{2}+1
$$

\footnotetext{
${ }^{22}$ Of course, this would not be the case if $K(t)$ were not a linear function of $p_{t-1}$. See Section 5 for a discussion.
} 
which as shown in Section 6.2 is satisfied when $r$ is sufficiently large. In particular, there exists a $r_{2}>r_{1}$ such if $T_{2}<T_{1}$ then it must be that $r>r_{2}$. Moreover, for having a large drop in the amount of new investors, we need $\alpha_{L}$ sufficiently low. Specifically, we need

$$
\alpha_{L}<\alpha_{L}^{*}
$$

where $\alpha_{L}^{*}$ is defined in (19). As shown in Section 6.1, if $T=T_{2}$ and $\alpha_{L}<\alpha_{L}^{*}$ then

$$
p_{T+1}^{I, T+1}<p_{T} .
$$

That is, upon observing that the price has not increased due to the massive sale of rational investors, $I$-investors become aware that they had overestimated the duration of the bubble and that the price will drop at $T+1$ and never recover. We can summarize our results in the following Proposition.

Proposition 3 There exist $r_{1}>0$ and $r_{2}>r_{1}$ such that, upon observing the exit of $R$-agents at $T, I$-agents realize that future prices will be lower than expected only if

$$
r>r_{1},
$$

and they realize that the bubble will be shorter than expected only if

$$
r>r_{2} .
$$

\section{Uncertainty Aversion}

In this section we extend our basic model to allow for uncertainty aversion. ${ }^{23}$ In our setting, uncertainty concerns solely the predictions of other investors' strategies (otherwise, the underlying dynamics is deterministic). Hence, the amount of uncertainty faced by each agent depends on his ability to understand these strategies. Rational agents face no uncertainty given that they

\footnotetext{
${ }^{23}$ Uncertainty (or equivalently ambiguity) describes situations in which agents' perceptions need not be accurate enough to provide them with a unique probability measure over the possible states of the world.
} 
have perfect understanding. But, $I$-agents face an uncertain environment given that they only know coarse statistics about others' investment attitudes. When an $I$-agent is sufficiently uncertainty averse, he may refrain from investing.

We will describe how to extend our basic model to cover such a situation with the aim of shedding new light on the relation between the magnitude of bubbles and the proportion of rational investors. This is a fundamental issue for the efficient market hypothesis, whereby rational investors play a stabilizing role and ensure market efficiency.

The efficient market hypothesis has been challenged by the behavioral finance literature, most notably by De Long et al. (1990b). In their model, the presence of extrapolative investors (who buy when the price has increased) induces rational investors to artificially increase the price and then sell at an even higher price to extrapolative agents. The higher the share of rational agents the higher are initial prices, the more distorted are extrapolative agents' expectations and so the larger is the size of the bubble.

In our basic model without uncertainty aversion, increasing the share of rational investors always reduces the duration of the maximal bubble (see Proposition 2), thereby suggesting a sharp contrast with the literature based on extrapolative agents. ${ }^{24}$ But, the relation between the maximal bubble and the share of rational investors can go the other way in the extension of our model to uncertainty aversion. The basic reason is that, even if uncertainty averse, fully rational agents may still be willing to invest since as noted they face no uncertainty. This is not the case for uncertainty-averse $I$-agents. As a result, the amount of investors in the speculative market is in general increasing with the share of rational agents, which may in turn induce more optimistic expectations and sustain longer bubbles. We think this paper is the first to highlight the role of uncertainty aversion in the relation between rational agents and bubbles.

In order to formally illustrate this idea, we assume that investors differ

\footnotetext{
${ }^{24}$ Of course, there are other models which make the same prediction as this one. In Abreu and Brunnermeier (2003), for example, rational investors stay invested longer the higher is the buying capacity of irrational agents; then, the lower the share of irrational investors the lower is their buying capacity and so the shorter is the bubble.
} 
in their attitudes towards ambiguity. Each agent irrespective of his cognitive type is ambiguity averse with probability $h$ and ambiguity neutral with probability $1-h$. Ambiguity aversion is not relevant for $R$-investors, however, since such investors face no ambiguity, as observed above. For $I$-investors, instead, let us denote by $H$ those who are ambiguity-averse and by $L$ those who are ambiguity neutral. The respective masses of $H$ and $L$ investors are $(1-r) h$ and $(1-r)(1-h)$. Admitting that their predictions can be mistaken by some $\varepsilon, H$-agents believe that, in every $t$, the actual buy rate $\beta_{t}$ will be in the interval $[\bar{\beta}-\varepsilon, \bar{\beta}+\varepsilon] \cap[0,1]$ and the actual sell rate $\sigma_{t}$ will be in the interval $[\bar{\sigma}-\varepsilon, \bar{\sigma}+\varepsilon] \cap[0,1] .{ }^{25}$ Furthermore, these agents choose the optimal investment strategy by considering the worst realizations of $\beta_{t}$ and $\sigma_{t}{ }^{26}$ Investors of type $L$ are instead neutral towards uncertainty (or, alternatively, they do not admit that their predictions can be mistaken). Hence, as in Section 3, such investors only consider the averages $\bar{\beta}$ and $\bar{\sigma}$.

We define an equilibrium similar to the one above in which $H$-agents stop investing as soon as they can (this requires $\varepsilon$ to be large enough), $R$-agents exit at some $T$, and $L$-agents exit at $T+1$, when the crash occurs. As above, the maximal duration of the bubble $T_{\max }$ is defined by two conditions: $L$ agents should buy at $T$, which defines $T_{1}$, and $p_{T} \geq p_{T-1}$, which defines $T_{2}$. As detailed in the Appendix, the effect of increasing $r$ on $T_{\max }$ depends on whether $T_{1}$ or $T_{2}$ binds. In fact, it can be shown that $T_{2}$ decreases with $r$, while $T_{1}$ increases in $r$ if and only if $T h>1$. Hence, provided that $h$ is not too small, $T_{\max }$ increases in $r$ when $T_{1}<T_{2}$. This is the case when $r$ is not too large. We state this more formally in the next Proposition.

Proposition 4 If $T h>1$, there exists a $\hat{r} \in(0,1)$ such that $T_{\max }$ increases in $r$ for every $r \leq \hat{r}$.

\footnotetext{
${ }^{25}$ The error term $\varepsilon$ is here taken as given. One could for example endogenize this interval by letting the expected $\beta_{t}$ and $\sigma_{t}$ lie between the minimum and the maximum buy and sell rates observed along the equilibrium.

${ }^{26}$ Formally, we are assuming that these investors have a set of probability measures over the possible realizations of $\beta_{t}$ and $\sigma_{t}$. Investors compute the minimal expected payoffs conditional on each possible prior, and decide the investment strategy corresponding to the maximum of such payoffs. This idea, which may be thought as an extreme form of uncertainty aversion, was formalized by Gilboa and Schmeidler (1989).
} 


\section{Discussion}

\subsection{Relation with empirical evidence}

Our equilibrium construction matches several empirical regularities of bubble episodes. First, bubbles are characterized not only by extreme price fluctuations but also by high volumes of trade (Cochrane, 2002; Hong and Stein, 2007). In our model, the volume of trade increases over time, and it reaches its peak just before the crash, when heterogeneity of expectations is highest. $^{27}$ Second, speculative stocks tend to be initially in short supply and bubbles are sustained by the large involvement of new investors (Cochrane, 2002; Kindleberger, 2005). This is consistent with our finding in Proposition 2 that bubbles are likely to be bigger when $\alpha_{H}$ is larger and/or when $X$ is smaller. Third, bubbles tend to display slow booms and sudden crashes (Veldkamp, 2005), which is consistent with the effect of a small $z$ as this implies that the selling pressure increases slowly.

Another fundamental ingredient of bubble episodes is that market participants become increasingly euphoric and then (some of them) realize they have been invested for too long and panic (Shleifer, 2000; Kindleberger, 2005). In our model, the same group of investors may switch from euphoria to panic along the equilibrium when rational investors are not too few. Such a finding thus requires the presence of both rational and irrational investors and we suspect that this may be a plausible feature of real bubbles. Indeed, real bubbles seem to attract a large number of inexperienced investors: for example, Greenwood and Nagel (2008) and Griffin, Harris, Shu and Topaloglu (2009) show that inexperienced investors sustained the Internet bubble (see also Kindleberger, 2005). This is in line with Proposition 1 which shows that $r$ should not be too large in such cases to make the emergence of bubbles possible. At the same time, as in the spirit of Proposition 3, it appears that rational investors too invest in real bubbles. Indeed, Brunnermeier and Nagel (2004) and Temin and Voth (2004) document how in various bubble episodes

\footnotetext{
${ }^{27}$ Moreover, while we here abstract from this mechanism, these volumes may in itself reinforce the bubble as investors tend to pay attention to stocks with abnormal trade volumes (see Barber and Odean, 2008).
} 
major investors earned large profits by timing the market correctly.

Last, by focusing on a setting in which information is complete but some people face limitations in processing all the relevant aspects of such information, we stress that information availability per se need not lead to market efficiency. Instead, we point out that information accessibility -which focuses on whether information is presented in a way that facilitates its interpretationshould matter as well. For example, our analysis may be suggestive of the idea that when financial advisors concentrate on aggregate statistics such as the long term profitability of some types of investments (as opposed to highlighting the more complete charts showing the life cycle of asset prices), there is a risk that less sophisticated investors extrapolate from this information in a way that makes the emergence of bubbles more likely. It may also be the case that some news have a destabilizing effect as it may lead partially sophisticated investors to get excessively excited. (This is in a sense what happens in our model when these investors observe unexpected increases in the price.) Indeed, a number of recent papers have documented the destabilizing role of media coverage on asset pricing (see among others Dyck and Zingales, 2003; Veldkamp, 2006; Tetlock, 2007; Bhattacharya, Galpin, Ray and $\mathrm{Yu}, 2009)$.

\subsection{Interpretation of the equilibrium}

The key methodological contribution of our approach is to offer an equilibrium perspective to the behavior of non-fully rational investors. Endogenizing the expectations of non-fully rational investors allows us to study how these depend on the strategic environment and at how they evolve along the bubble. We come back here to the interpretation of our equilibrium approach.

As often with equilibrium approaches, we think of it as the result of a process of learning. More precisely, we have in mind that over long periods, bubble episodes repeat periodically with new cohorts of investors. Investors interpret the current market in light of historical data about similar episodes, but investors may be heterogeneous in terms of which statistics they consider to analyze these data. While our fully rational investors analyze such data 
with elaborate statistics, which leads them to know $\beta_{t}$ and $\sigma_{t}$ and so to rightly understand the trade dynamics, partially sophisticated investors use a simplified model, able to provide the correct averages $\bar{\beta}$ and $\bar{\sigma}$ but no more detailed statistics. In a sense, they apply a linear model to analyze trade dynamics that are not necessarily linear (as in the spirit of Sargent, 1993).

It should be emphasized that we do not view $I$-investors as computing the averages $\bar{\beta}$ and $\bar{\sigma}$ from the sequence of $\beta_{t}$ and $\sigma_{t}$, which they are not assumed to know. They know the average buying and selling strategies, and no more, in the same way that some inexperienced investors in the real word are only informed that on average the stock market yields higher returns than the housing market but are not informed of the specific circumstances in which the converse relation may hold.

Given that $I$-investors know only $\bar{\beta}$ and $\bar{\sigma}$, there is no way in which, based on their historical knowledge, these investors could update their expectations about the future buying and selling strategies as a function of the history of trade. ${ }^{28}$ This is why, even when they observe realizations of $\beta_{t}$ and $\sigma_{t}$ which are different from the mean, $I$-agents keep expecting that future $\beta_{t}$ and $\sigma_{t}$ will match $\bar{\beta}$ and $\bar{\sigma}$. They may understand that they only know the average from which actual realizations may differ, but, as sketched in Section 4, this may not be enough to prevent the occurrence of bubbles. ${ }^{29}$

\footnotetext{
${ }^{28}$ In particular, as these strategies are all investors know, they need not know that there are different types of investors in the market. Hence, $I$-agents are not aware that other investors may have a more accurate understanding of the market dynamics. Otherwise, given that in our model trades mostly occur for speculative reasons, $I$-agents may simply decide to stay outside the market if they realize they are less sophisticated than others.

${ }^{29}$ One may also think of other reasons for why $I$-agents do not update their model about the underlying distribution of strategies. For example, they may perceive that some stochastic element, specific to the current bubble, affects both demand and supply. It is not difficult to accommodate our model to incorporate extra randomness, and in this case any realization of prices and trade volumes would be possibly compatible with the expected $\bar{\beta}$ and $\bar{\sigma}$. As another alternative, assuming agents adjust their theory when it is proven sufficiently wrong, agents in our model get a strong enough signal that their trading decisions are wrong only when it is too late.
} 


\subsection{Robustness and extensions}

This paper has offered a framework to analyze speculative bubbles in an equilibrium fashion in which investors differ in their cognitive abilities. The key mistake partially sophisticated investors make is in predicting when other investors stop buying and so when the crash occurs. As already argued, such a prediction is likely not to be accurate as abrupt changes in investors' strategies tend to occur even with no major news, in days quite similar to days in which most investors wanted to buy. Nonetheless, I-investors' expectation of constant buy and sell rates throughout the bubble episode may be too extreme as well. While we leave for future research the task of exploring more systematically alternative cognitive assumptions, we suspect that our results will be robust to broader specifications of the cognitive types.

Suppose for illustration that partially sophisticated investors distinguish two phases of the bubble: they expect some $\bar{\beta}$ and $\bar{\sigma}$ to occur in each $t<$ $t^{*}$ and some other $\bar{\beta}^{\prime}$ and $\bar{\sigma}^{\prime}$ to occur at each $t \geq t^{*}$, where as above these expectations correspond to the true average strategies played within each stage of the bubble. Consider the bubble equilibrium described in equation (6) and suppose $t^{*} \leq T$. During the early stage of the bubble, $I$-agents' expectation is correct since strategies are indeed constant. The expectation in the second stage of the bubble depends on $t^{*}$. The higher is $t^{*}$ the more precisely late strategies are perceived. In particular, the higher $t^{*}$, the less optimistic is $I$-agents' expectation and so the lower is the maximal sustainable bubble. However, to the extent that the strategies played in the last period of the bubble are perceived with some error, i.e. $t^{*} \leq T$, one can define some parameter values such that a bubble equilibrium exists.

Another extension worth exploring is on the characteristics of the demand and supply that would increase the magnitude of bubbles. Regarding the amount of new investors attracted in every period $t$, we have previously considered a (piecewise) linear $K_{t}$ (in $p_{t-1}$ ), which simplified the analysis and made optimal investment strategies independent on the currently realized price. The assumption that $\alpha_{L} \leq \alpha_{H}$, allows to express in a simple way how the exit of rational investors represents a bad shock for other investors' 
expectation. As shown in Proposition 3, when $r>r_{2}$ and $\alpha_{L}<\alpha_{L}^{*}$, the exit of rational investors at $T$ is sufficient to induce $I$-investors to believe that the crash will occur at $T+1$. This makes our result on the emergence of bubbles even stronger, since in this case rational investors would always be in the position to successfully attack the bubble (while at the same time having the incentive not to attack it immediately, as in Abreu and Brunnermeier, 2003).

On the other hand, the possibility of having an endogenous crash in our framework does not crucially depend on $\alpha_{L}$. Even if $T_{2}<T_{1}$ and $\alpha_{L}=\alpha_{H}$, $R$-investors could sell gradually between $T_{2}$ and $T_{1}$ and the crash would occur at $T_{1}+1$ (thereby making the crash somewhat less sudden). Equation (6), and the subsequent computations of (3), would be slightly modified, but the substance of our analysis would remain valid.

More generally, the functional form of $K_{t}$ determines the relation between market trends and expectations dynamics, as analyzed in Section 3.3. If $K_{t}$ is increasing, a price higher than expected always leads $I$-investors to expect even higher prices in the future. Their corresponding expectation about the duration of the bubble, instead, depends on the curvature of $K_{t}$. If $K_{t}$ is convex (concave), an higher price than expected moves the expected date of the crash upwards (downwards). Accordingly, if $K_{t}$ is convex (concave), optimal strategies defined in (5) would take the form of stop (limit) orders, whereby investors buy if the price falls above (below) a given threshold. While the analysis would be more complicated, the spirit of our exercise would survive in that, given different expectations about other investors' strategies, $I$ investors and $R$-investors would typically employ different threshold. These differences in opinion may lead to fluctuations in the price which can then be exploited by rational investors. Getting more precise information about the shape of $K_{t}$ would allow to test the link between market trends and how expectations evolve in the life cycle of speculative markets.

Finally, as another extension, one may consider agents who would base their trading strategies on aggregate statistics other than the ones considered in this paper. For example, one such statistics could be the aggregate price growth rates. In this case, assuming agents somehow know that the price cannot go beyond some threshold, we could generate bubble dynamics similar 
to the ones found above (with investors updating their expectations about future prices upon observing current price realizations). Thus, for some statistical knowledge, the dynamics would be rather similar to the one found in our model. For other statistics, though, the analysis would be rather different. For example, if investors considered average prices at the peak of the bubble or average durations of the bubble, there would be no way in which they could update their expectations upon the observation of current market trends. At the same time, in a completely deterministic model as above, this knowledge would prevent the emergence of bubbles (since the duration of past bubbles would be the same as in the current bubble). Our analysis has considered learning of investment strategies as we believe the fundamental issue that investors face in real bubble episodes lies in understanding how other investors' strategies evolve. ${ }^{30}$ Nonetheless, a more systematic investigation of how different types of learning affect investment decisions and the functioning of the market remains in our view a very interesting avenue for future research.

\section{References}

Abreu, Dilip and Markus K. Brunnermeier, "Bubbles and Crashes," Econometrica, 2003, 71 (1), 173-204.

Allen, Franklin and Douglas Gale, "Limited Market Participation and Volatility of Asset Prices," American Economic Review, September 1994, 84 (4), 933-55.

— and _ _ "Bubbles and Crises," Economic Journal, January 2000, 110 (460), 236-55.

\footnotetext{
${ }^{30}$ Knowing the past realizations of $T$ or of the maximum trading price may be of little guidance for predicting the dynamics of the current bubble. These statistics crucially depend on the specificities of a given bubble episode (in terms for example of markets involved, asset supply and investment capacity) and as such they are unlikely to stabilize. By contrast, understanding investment strategies allows agents to update their expectations about future prices based on current specificities and market trends and so equip them with a better tool for understanding the current bubble.
} 
, Stephen Morris, and Andrew Postlewaite, "Finite Bubbles with Short Sale Constraints and Asymmetric Information," Journal of Economic Theory, 1993, 61 (2), 206-229.

Barber, Brad M. and Terrance Odean, "All That Glitters: The Effect of Attention and News on the Buying Behavior of Individual and Institutional Investors," Review of Financial Studies, April 2008, 21 (2), 785-818.

Bhattacharya, Utpal, Neal Galpin, Rina Ray, and Xiaoyun Yu, "The Role of the Media in the Internet IPO Bubble," Journal of Financial and Quantitative Analysis, June 2009, 44 (03), 657-682.

Biais, Bruno and Peter Bossaerts, "Asset Prices and Trading Volume in a Beauty Contest," Review of Economic Studies, April 1998, 65 (2), $307-40$.

Bianchi, Milo and Philippe Jehiel, "Bubbles and Crashes with Partially Sophisticated Investors," Paris School of Economics Working Paper No. 2008-62 2008.

Brunnermeier, Markus K., "Bubbles," in Steven Durlauf and Lawrence Blume, eds., The New Palgrave Dictionary of Economics, New York: Oxford University Press, 2007.

and Stefan Nagel, "Hedge Funds and the Technology Bubble," Journal of Finance, October 2004, 59 (5), 2013-2040.

Camerer, Colin F., Teck-Hua Ho, and Juin-Kuan Chong, "A Cognitive Hierarchy Model of Games," Quarterly Journal of Economics, August 2004, 119 (3), 861-898.

Cochrane, John H., "Stocks as Money: Convenience Yield and the TechStock Bubble," NBER Working Paper No. 8987 June 2002.

Cutler, David M., James M. Poterba, and Lawrence H. Summers, "What Moves Stock Prices?," Journal of Portfolio Management, Spring 1989, $15(3), 4-12$. 
$\ldots$, , and "Speculative Dynamics and the Role of Feedback Traders," American Economic Review, May 1990, 80 (2), 63-68.

De Long, J. Bradford, Andrei Shleifer, Lawrence H. Summers, and Robert J. Waldmann, "Noise Trader Risk in Financial Markets," Journal of Political Economy, 1990, 98 (4), 703-38.

$\ldots$ _ _ _ _ , and _ _ , "Positive Feedback Investment Strategies and Destabilizing Rational Speculation," Journal of Finance, June 1990, 45 (2), 379-395.

Dyck, Alexander and Luigi Zingales, "The Media and Asset Prices," Mimeo: Harvard Business School and University of Chicago 2003.

Eyster, Erik and Matthew Rabin, "Cursed Equilibrium," Econometrica, 09 2005, 73 (5), 1623-1672.

Garber, Peter M., "Famous First Bubbles," Journal of Economic Perspectives, 1990, 4 (2), 35-54.

Gennotte, Gerard and Hayne Leland, "Market Liquidity, Hedging, and Crashes," American Economic Review, December 1990, 80 (5), 9991021.

Gilboa, Itzhac and David Schmeidler, "Maxmin Expected Utility with Nonunique Prior," Journal of Mathematical Economics, 1989, 18, 141153.

Greenwood, Robin and Stefan Nagel, "Inexperienced Investors and Bubbles," NBER Working Paper No. 14111 June 2008.

Griffin, John M., Jeffrey H. Harris, Tao Shu, and Selim Topaloglu, "Who Drove and Burst the Tech Bubble?," McCombs Research Paper 2009

Haruvy, Ernan, Yaron Lahav, and Charles N. Noussair, "Traders' Expectations in Asset Markets: Experimental Evidence," American Economic Review, December 2007, 97 (5), 1901-1920. 
Higgins, E. Tory, "Knowledge activation: Accessibility, applicability and salience.," in E. Tory Higgins and Arie E. Kruglanski, eds., Social psychology: Handbook of basic principles, New York: Guilford., 1996, pp. 133-168.

Hirshleifer, David, "Investor Psychology and Asset Pricing," Journal of Finance, 2001, 56 (4), 1533-1597.

Hong, Harrison and Jeremy C. Stein, "Disagreement and the Stock Market," Journal of Economic Perspectives, Spring 2007, 21 (2), 109128.

_ _ José Scheinkman, and Wei Xiong, "Asset Float and Speculative Bubbles," Journal of Finance, 06 2006, 61 (3), 1073-1117.

Jehiel, Philippe, "Limited horizon forecast in repeated alternate games," Journal of Economic Theory, 1995, 67, 497-519.

_ , "Analogy-based expectation equilibrium," Journal of Economic Theory, 2005, 123 (2), 81-104.

and Dov Samet, "Valuation Equilibria," Theoretical Economics, 2007, 2, 163-185.

Kahneman, Daniel, "Maps of Bounded Rationality: Psychology for Behavioral Economics," American Economic Review, December 2003, 93 (5), 1449-1475.

_ and Amos Tversky, "Prospect Theory: An Analysis of Decision under Risk," Econometrica, March 1979, 47 (2), 263-91.

Kindleberger, Charles, Manias, panics, and crashes: a history of financial crises, Wiley and Sons, 2005.

Koszegi, Botond and Matthew Rabin, "A Model of ReferenceDependent Preferences," Quarterly Journal of Economics, November 2006, 121 (4), 1133-1165. 
Milgrom, Paul and Nancy Stokey, "Information, trade and common knowledge," Journal of Economic Theory, February 1982, 26 (1), 1727.

Morris, Stephen, "Speculative Investor Behavior and Learning," Quarterly Journal of Economics, November 1996, 111 (4), 1111-33.

Nagel, Rosemarie, "Unraveling in Guessing Games: An Experimental Study," American Economic Review, December 1995, 85 (5), 1313-26.

Porter, David P. and Vernon L. Smith, "Stock Market Bubbles in the Laboratory," Journal of Behavioral Finance, 2003, 4 (1), 7-20.

Rubinstein, Ariel, Modeling Bounded Rationality, The MIT Press, 1998.

Sargent, Thomas, Bounded Rationality in Macroeconomics, Oxford University Press, 1993.

Scheinkman, Jose A. and Wei Xiong, "Overconfidence and Speculative Bubbles," Journal of Political Economy, 2003, 111 (6), 1183-1219.

Shiller, Robert J., Market Volatility, Cambridge MA: MIT Press, 1989.

__ , "Measuring Bubble Expectations and Investor Confidence," Journal of Psychology and Financial Markets, 2000, 1 (1), 49-60.

Shleifer, Andrei, Inefficient Markets, Oxford University Press, 2000.

Stahl, Dale O. and Paul W. Wilson, "On Players' Models of Other Players: Theory and Experimental Evidence," Games and Economic Behavior, July 1995, 10 (1), 218-254.

Stiglitz, Joseph E., "Symposium on Bubbles," Journal of Economic Perspectives, 1990, 4 (2), 13-18.

Temin, Peter and Hans-Joachim Voth, "Riding the South Sea Bubble," American Economic Review, December 2004, 94 (5), 1654-1668. 
Tetlock, Paul C., "Giving Content to Investor Sentiment: The Role of Media in the Stock Market," Journal of Finance, 06 2007, 62 (3), 11391168.

Tirole, Jean, "On the Possibility of Speculation Under Rational Expectations," Econometrica, 1982, 50 (5), 1163-1182.

Veldkamp, Laura, "Slow boom, sudden crash," Journal of Economic Theory, October 2005, 124 (2), 230-257.

_ _ "Media Frenzies in Markets for Financial Information," American Economic Review, June 2006, 96 (3), 577-601. 


\section{Omitted proofs}

\subsection{Proof of Proposition 2}

Observe first that since $\left(\beta_{t}, \sigma_{t}\right)=(1, z)$ for $t \leq T-1$ and $T<T_{0}$, we have that $p_{t-1}>p_{t-2}$ and so by (13) $K_{t}=\alpha_{H} p_{t-1}$ for $t \leq T$. Hence, condition (9) writes as

$$
\frac{\bar{\beta} \alpha_{H} p_{t}^{I, t}}{\bar{\sigma}(t+1) X} \geq p_{t}^{I, t} \Leftrightarrow t+1 \leq \frac{\bar{\beta}}{\bar{\sigma}} \frac{\alpha_{H}}{X} \text { for each } t \leq T .
$$

In particular, (21) must hold for $t=T$. Substituting $\bar{\beta}$ and $\bar{\sigma}$ with (7) and (8), we have

$$
T+1 \leq \frac{T-r}{T z+r(1-z)+1} \frac{\alpha_{H}}{X} \equiv W(T),
$$

where it can be shown with simple algebra that $W(T)$ is increasing and concave in $T$. Defining $T_{1}$ as the largest $T$ such that $T+1=W(T)$, condition (9) defines an upper bound on $T$ as expressed in (16) in the text.

Turning to condition (10), we need to distinguish two cases, depending on whether or not $p_{T+1}^{I, T+1}$ exceeds $p_{T}$. Suppose first that $p_{T+1}^{I, T+1}>p_{T}$ and so $K_{T+2}^{I, T+1}=\alpha_{H} p_{T+1}^{I, T+1}$. Then, condition (10) writes

$$
\frac{\bar{\beta} \alpha_{H} p_{T+1}^{I, T+1}}{\bar{\sigma}(T+2) X}<p_{T+1}^{I, T+1} \Leftrightarrow T+2>W(T) .
$$

Similarly to above, let $T_{3}$ be the largest $T$ such that $T+2=W(T)$. Then, condition (10) defines a lower bound on $T$ such that

$$
T>T_{3},
$$

where it is clear from (15) and (23) that $T_{3}<T_{1} \cdot{ }^{31}$ Suppose instead that $p_{T+1}^{I, T+1} \leq p_{T}$ and so $K_{T+2}^{I, T+1}=\alpha_{L} p_{T+1}^{I, T+1}$. Condition (10) now writes

$$
T+2>\frac{T-r}{T z+r(1-z)+1} \frac{\alpha_{L}}{X} \equiv \widetilde{W}(T)
$$

\footnotetext{
${ }^{31}$ Notice also that if $t$ were continuous we would have $T_{1}=T_{3}$ so $T$ would be pinned down by conditions (15) and (23) when $p_{T+1}^{I, T+1}>p_{T}$.
} 
As before, let $\tilde{T}_{3}$ be the largest $T$ such that $T+2=\widetilde{W}(T)$. Then, condition (10) defines a lower bound on $T$ such that

$$
T>\tilde{T}_{3}
$$

where $\tilde{T}_{3} \leq T_{3}$ follows from $\alpha_{L} \leq \alpha_{H}$. From equations (23) and (25), it also clear that if $I$-investors sell at $T+1$ then they do not buy at any later point in time.

We can now derive more formally when $T_{\max }$ can be sustained as equilibrium. If $T_{1} \leq T_{2}, T_{\max }$ is an equilibrium if $T_{1} \geq 1$ and if $I$-investors sell at $T_{1}+1$. Given the shape of the function $W(T)$ described in $(22), W(T)$ and $T+1$ intersects once and only once when $W(T) \geq T+1$ for $T=1$. This is used to derive condition (18) in the text, which ensures that $T_{1} \geq 1$. Given that as noticed $T_{1}>T_{3} \geq \tilde{T}_{3}$, condition (10) is satisfied at $T_{1}$ so indeed $I$-investors sell at $T_{1}+1$. If $T_{2}<T_{1}, T_{\max }$ is an equilibrium if $I$-investors sell at $T_{2}+1$, that is if

$$
p_{T_{2}+2}^{I, T_{2}+1}<p_{T_{2}+1}^{I, T_{2}+1}
$$

To see when (27) holds, suppose first that $p_{T_{2}+1}^{I, T_{2}+1}>p_{T_{2}}$, then (27) writes

$$
\frac{\bar{\beta} \alpha_{H}}{\bar{\sigma}\left(T_{2}+2\right) X}<1,
$$

which is not satisfied unless $T_{2}>T_{3}$. Hence, in general, we need that

$$
p_{T_{2}+1}^{I, T_{2}+1} \leq p_{T_{2}}
$$

Notice that since $p_{T_{2}}=p_{T_{2}-1},(28)$ writes

$$
\frac{\bar{\beta} \alpha_{L}}{\bar{\sigma}\left(T_{2}+1\right) X}<1,
$$

that is

$$
\alpha_{L}<\frac{\bar{\sigma}}{\bar{\beta}}\left(T_{2}+1\right) X \equiv \alpha_{L}^{*}(r, z, X)
$$

which is condition (19) in the text. If (19) holds, then (28) holds and (27) 
writes

$$
\frac{\bar{\beta} \alpha_{L}}{\bar{\sigma}\left(T_{2}+2\right) X}<1
$$

The last inequality is implied by (19), which is then a sufficient condition for $T_{2}$ to be an equilibrium. Finally, notice that $\alpha_{L}^{*}>0$ and $\alpha_{L}^{*}<\alpha_{H}$ since by construction

$$
\alpha_{H}=\frac{\bar{\sigma}}{\bar{\beta}}\left(T_{1}+1\right) X,
$$

and we are considering the case $T_{2}<T_{1}$.

Turning to the second part of the Proposition, to see that $T_{1}$ increases in $\alpha_{H}$ and decreases with $X, z$ and $r$ recall that $T_{1}$ is defined by $T+1=W(T)$ and notice with simple algebra that $W(T)$ increases in $\alpha_{H}$ and decreases with $X, z$ and $r$ for all $T$. The fact that $T_{2}$ increases in $\alpha_{H}$ and decreases with $X$, $z$ and $r$ can be derived by simple differentiation of equation (17).

\subsection{Proof of Proposition 3}

We first show that there exists a $r_{1}>0$ such that for (20) to be satisfied we need $r>r_{1}$. Notice that $p_{T+1}^{I, T+1}<p_{T+1}^{I, T}$ is equivalent to

$$
\frac{\bar{\beta} K_{T+1}}{\bar{\sigma} X_{T+1}}<\frac{\bar{\beta} K_{T+1}^{I, T}}{\bar{\sigma} X_{T+1}},
$$

that is $K_{T+1}<K_{T+1}^{I, T}$. Given (13), this is equivalent to

$$
p_{T}=\frac{\beta_{T} K_{T}}{\sigma_{T} X_{T}}<p_{T}^{I, T}=\frac{\bar{\beta} K_{T}}{\bar{\sigma} X_{T}}
$$

that is

$$
\frac{\beta_{T}}{\sigma_{T}}<\frac{\bar{\beta}}{\bar{\sigma}}
$$

With simple algebra, it can be shown that condition (29) is satisfied if and only if $\operatorname{Tr}>1$, which is condition (20) in the text. In particular, recall that we must have $T \leq T_{2}$, so $r T_{2}>1$. Doing the algebra, the last inequality is satisfied (if ever) for $r \in\left(r_{1}, r_{1}^{\prime}\right)$, where $r_{1}>0$.

We then show that there exists a $r_{2}>0$ such that if $T_{2}<T_{1}$ then it 
must be that $r>r_{2}$. Notice first that since by definition $W(T)>T+1$ for $T<T_{1}, T_{2}<T_{1}$ writes

$$
W\left(T_{2}\right)>T_{2}+1
$$

Notice that for $r=0$,

$$
T_{2}=\frac{\alpha_{H}}{z X},
$$

and since, from (15), $W(T)$ is bounded above by $\alpha_{H} / z X$, then (30) is not satisfied for $r=0$. Moreover, both $T_{2}$ and $W\left(T_{2}\right)$ are decreasing in $r$. Doing the algebra, (30) is satisfied (if ever) for $r \in\left(r_{2}, r_{2}^{\prime}\right)$, where $r_{2}>0$.

Finally, to see that $r_{2}>r_{1}$, notice that by definition of $T_{2}$ and $W\left(T_{2}\right)$, (30) writes

$$
\frac{\bar{\beta}}{\bar{\sigma}} \frac{\alpha_{H}}{X}>\frac{\beta_{T}}{\sigma_{T}} \frac{\alpha_{H}}{X}+1 .
$$

Hence, condition (29) is necessary but not sufficient for (30).

\subsection{Proof of Proposition 4}

We consider an equilibrium where (for simplicity) $H$-investors perceive enough uncertainty to be induced to stop investing as soon as they can (for example, they understand that $\beta_{t}$ and $\sigma_{t}$ are drawn by distributions with support on $[0,1]$ and since they are extremely ambiguity-adverse they assume $\beta_{t}=0$ and $\sigma_{t}=1$ for all $t$ ). $R$-agents exit at some $T$, and $L$-agents exit at $T+1$, when the crash occurs (we use the same notation about $T$ than in the previous analysis even thought the variables are obviously not the same unless $h=0$ ). The resulting equilibrium buy and sell rates are

$$
\left(\beta_{t}, \sigma_{t}\right)=\left\{\begin{array}{cl}
(1-(1-r) h, z+(1-z)(1-r) h) & \text { for } t \leq T-1 \\
((1-r)(1-h), z+(1-z)(r+(1-r) h)) & \text { for } t=T \\
(0,1) & \text { for } t=T+1
\end{array}\right.
$$

The maximal duration of the bubble $T_{\max }$ is defined by two conditions: $L$ agents should buy at $T$, that is

$$
T+1 \geq \frac{\bar{\beta}}{\bar{\sigma}} \frac{\alpha_{H}}{X}
$$


which defines $T_{1}$, where $T_{1}$ increases in the ratio $\bar{\beta} / \bar{\sigma}$. I-agents are induced to expect the buy and sell rates

$$
\bar{\beta}=\frac{(1-h) T+r(T h-1)}{T+1},
$$

and

$$
\bar{\sigma}=\frac{1+T z+T(1-z) h-r(1-z)(T h-1)}{T+1} .
$$

From the above expression it is clear that $\bar{\beta} / \bar{\sigma}$ increases in $r$ if and only if $T h>1$, so $T_{1}$ increases in $r$ if and only if $T h>1$. The second condition is that $p_{T} \geq p_{T-1}$, which defines

$$
T_{2} \equiv \frac{(1-r)(1-h)}{z+(1-z)(r+(1-r) h)} \frac{\alpha_{H}}{X}
$$

which is decreasing in $r$. Hence, if $T h>1, T_{\max } \equiv \min \left\{T_{1}, T_{2}\right\}$ increases in $r$ when $T_{1}<T_{2}$. To see when this is the case, notice that at $r=0, T_{1}<T_{2}$ since $T_{2}+1>W\left(T_{2}\right)$. In fact for $T=T_{2}$

$$
T+1>\frac{\bar{\beta}}{\bar{\sigma}} \frac{\alpha_{H}}{X}=\frac{(1-h) \frac{\alpha_{H}}{X}}{1+(1-h) \frac{\alpha_{H}}{X}} T .
$$

Notice also that at $r=1, T_{1}>0=T_{2}$. Since for $T h>1, T_{2}$ decreases with $r$ while $T_{1}$ increases in $r$, there exists a unique $\hat{r} \in(0,1)$ such that $T_{1}<T_{2}$ for every $r \leq \hat{r}$, that is $T_{\max }$ increases in $r$ for every $r \leq \hat{r}$. 